$0 \mathrm{pt}=0 \mathrm{pt}$

\title{
ON THE GENERALIZED FUGLEDE-PUTNAM THEOREM
}

\author{
M. H. M. RASHID, M. S. M. NOORANI AND A. S. SAARI
}

\footnotetext{
Abstract. In this paper, we prove the following assertions:

(1) If the pair of operators $\left(A, B^{*}\right)$ satisfies the Fuglede-Putnam Property and $S \in \operatorname{ker}\left(\delta_{A, B}\right)$, where $S \in \mathbf{B}(\mathscr{H})$, then we have

$$
\left\|\delta_{A, B} X+S\right\| \geq\|S\| .
$$

(2) Suppose the pair of operators $\left(A, B^{*}\right)$ satisfies the Fuglede-Putnam Property. If $A^{2} X=X B^{2}$ and $A^{3} X=X B^{3}$, then $A X=X B$.

(3) Let $A, B \in \mathbf{B}(\mathscr{H})$ be such that $A, B^{*}$ are $p$-hyponormal. Then for any $X \in \mathscr{C}_{2}, A X-X B \in \mathscr{C}_{2}$ implies $A^{*} X-$ $X B^{*} \in \mathscr{C}_{2}$.

(4) Let $T, S \in \mathbf{B}(\mathscr{H})$ be such that $T$ and $S^{*}$ are quasihyponormal operators. If $X \in \mathbf{B}(\mathscr{H})$ and $T X=X S$, then $T^{*} X=X S^{*}$.
}

\section{Introduction}

Let $\mathscr{H}$ denote a separable, infinite dimensional Hilbert space. Let $\mathbf{B}(\mathscr{H}), \mathbf{C}_{2}$ and $\mathbf{C}_{1}$ denote the algebra of all bounded operators acting on $\mathscr{H}$, the Hilbert-Schmidt class and the trace class in $\mathbf{B}(\mathscr{H})$ respectively. It is known that $\mathbf{C}_{2}$ and $\mathbf{C}_{1}$ each form a two-sided ${ }^{*}-$ ideal in $\mathbf{B}(\mathscr{H})$ and $\mathbf{C}_{2}$ is itself a Hilbert space with inner product

$$
\langle X, Y\rangle=\sum\left\langle X e_{i}, Y e_{i}\right\rangle=\operatorname{tr}\left(X Y^{*}\right),
$$

where $\left\{e_{i}\right\}$ is any orthonormal basis of $\mathscr{H}$ and $\operatorname{tr}($.$) is the natural trace on \mathbf{C}_{1}$. The HilbertSchmidt norm of $X \in \mathscr{C}_{2}$ is given by $\|X\|_{2}$.

For operators $A, B \in \mathbf{B}(\mathscr{H})$, the generalized derivation $\delta_{A, B}(X)$ as an operator on $\mathbf{B}(\mathscr{H})$ is defined as follows:

$$
\delta_{A, B}(X)=A X-X B
$$

for all $X \in \mathbf{B}(\mathscr{H})$. When $A=B$, we simply write $\delta_{A}$ for $\delta_{A, A}$.

In [3], Anderson proved that if $N \in \mathbf{B}(\mathscr{H})$ is normal, $S$ is an operator such that $N S=S N$, then for all $X \in \mathbf{B}(\mathscr{H})$

$$
\left\|\delta_{N} X+S\right\| \geq\|S\|
$$

Received April 30, 2007.

2000 Mathematics Subject Classification. 47A10, 47B20, 47B47, 47A30.

Key words and phrases. Normal derivation, quasihyponormal, Hilbert Schmidt operator, $p$-hyponormal, Fuglede-Putnam Theorem. 
where $\|$.$\| is the usual operator norm. Thus in the sense of [3, Definition 1.2], Anderson's result$ says that the range of $\delta_{N}$ is orthogonal to the kernel of $\delta_{N}$, which is just the commutant $\{N\}^{\prime}$ of $N$.

Kittaneh [10] extended this result to an arbitrary unitarily invariant norm (for more information about this norm the reader should refer to [10]), and he proved the following theorem.

Theorem 1.1. If the operator $A, B \in \mathbf{B}(\mathscr{H})$ are normal, then for all $X, S \in \mathbf{B}(\not{H})$ such that $A S=S B$ we have

$$
|||A X-X B+S||| \geq|||S|||
$$

Recall that an operator $T$ in $\mathbf{B}(\mathscr{H})$ is called normal if $T^{*} T=T T^{*}$ and $p$-hyponormal if $\left(T^{*} T\right)^{p} \geq\left(T T^{*}\right)^{p}$, where $0<p \leq 1$. In particular 1-hyponormal is called hyponormal and $\frac{1}{2}$-hyponormal is called semi-hyponormal. The Löwner-Heinz inequality implies that if $T$ is $p$-hyponormal, then it is $q$-hyponormal for any $0<q \leq p$. Let $T=U|T|$ be the polar decomposition of $T$, where $U$ is partial isometry. Then $|T|$ is a positive square root of $T^{*} T$ and $\operatorname{ker} T=\operatorname{ker}|T|=\operatorname{ker} U$, where $\operatorname{ker}(T)$ denotes the kernel of $T$. Aluthge [2] introduced the operator $\widetilde{T}=|T|^{\frac{1}{2}} U|T|^{\frac{1}{2}}$ which is called the Aluthge transform, and also shown to satisfy the following result.

Theorem 1.2. Let $A=U|A|$ be the polar decomposition of a $p$-hyponormal for $0<p<1$ and $U$ is unitary. Then the following assertion holds:

(1) $\widetilde{A}=|A|^{\frac{1}{2}} U|A|^{\frac{1}{2}}$ is $p+\frac{1}{2}$-hyponormal if $0<p<\frac{1}{2}$.

(2) $\widetilde{A}=|A|^{\frac{1}{2}} U|A|^{\frac{1}{2}}$ is hyponormal if $\frac{1}{2} \leq p<1$.

According to [13], $T \in \mathbf{B}(\mathscr{H})$ is called dominant if $\operatorname{ran}(T-z I) \subseteq \operatorname{ran}(T-z I)^{*}$, for all $z \in$ $\sigma(T)$, where $\operatorname{ran}(T)$ and $\sigma(T)$ denotes the range and the spectrum of $T$.

It has been shown in [4, Theorem 3.4] that if $A, B^{*}$ and $S$ are operators in $\mathbf{B}(\mathscr{H})$ such that $B^{*}$ is $p$-hyponormal or log-hyponormal, $A$ is dominant and $A S=S B$, then for all $X \in \mathbf{B}(\mathscr{H})$ we have

$$
\|A X-X B+S\| \geq\|S\| .
$$

\section{Main Results}

We begin by the following definition of the orthogonality in the sense of Anderson [3, Definition 1.2] which generalize the idea of orthogonality in Hilbert space.

Definition 2.1. Let $\mathbb{C}$ be the field of complex numbers and let $X$ be a normed linear space. If $\|x-\lambda y\| \geq\|\lambda y\|$ for all $\lambda \in \mathbb{C}$ whenever $x, y \in X$, then $x$ is said to be orthogonal to $y$. Let $U$ and $W$ be two subspace in $X$. If $\|x+y\| \geq\|y\|$, for all $x \in U$ and for all $y \in W$, then $U$ is said to be orthogonal to $W$. 
Definition 2.2. Let $A, B \in \mathbf{B}(\mathscr{H})$. We say that the pair $\left(A, B^{*}\right)$ satisfies the Fuglede-Putnam Property, if whenever $S \in \operatorname{ker}\left(\delta_{A, B}\right)$, where $S \in \mathbf{B}(\mathscr{H})$ implies that $S \in \operatorname{ker}\left(\delta_{A^{*}, B^{*}}\right)$.

Theorem 2.3. Let $A, B, X \in \mathbf{B}(\mathscr{H})$. If the pair of operators $\left(A, B^{*}\right)$ satisfies the FugledePutnam Property and $S \in \operatorname{ker}\left(\delta_{A, B}\right)$, where $S \in \mathbf{B}(\mathcal{H})$ then we have

$$
\left\|\delta_{A, B} X+S\right\| \geq\|S\| .
$$

Proof. Since the pair $\left(A, B^{*}\right)$ satisfies the Fuglede-Putnam Property, it follows that $\overline{r a n S}$ reduces $A, \operatorname{ker}^{\perp} S$ reduces $B$. Let $A_{1}=\left.A\right|_{\overline{\text { ranS }}}, B_{1}=\left.B\right|_{\operatorname{ker}^{\perp} S}$ and let $S_{1}: \operatorname{ker}^{\perp} S \longrightarrow \overline{\text { ranS }}$ be the quasi-affinity defined by setting $S_{1} x=S x$ for each $x \in \operatorname{ker}^{\perp} S$. Then $\delta_{A_{1}, B_{1}}\left(S_{1}\right)=0=\delta_{A_{1}^{*}, B_{1}^{*}}\left(S_{1}\right)$, and it follows that $0 \notin \sigma\left(A_{1}\right)$ and $0 \notin \sigma\left(B_{1}\right)$.

Since the pair $\left(A, B^{*}\right)$ satisfies the Fuglede-Putnam Property, then $\delta_{A, B}(A S)=0$ implies $A_{1}^{*} B_{1}^{*} S_{1}^{*}=$ $A_{1} S_{1} B_{1}^{*-1}=A_{1} A_{1}^{*} S_{1}$, then $A_{1}$ is normal, similarly $B_{1}$ is normal.

Then, with respect to the orthogonal decompositions $\mathscr{H}=\overline{\operatorname{ranS}} \oplus(\overline{\text { ranS }})$ and $\mathscr{H}=\operatorname{ker}^{\perp} S \oplus$ ker $S, A$ and $B$ can be respectively represented as $A=\left(\begin{array}{cc}A_{1} & 0 \\ 0 & A_{2}\end{array}\right), B=\left(\begin{array}{cc}B_{1} & 0 \\ 0 & B_{2}\end{array}\right)$.

Now assume that the operators $S, X: \operatorname{ker}^{\perp} S \oplus \operatorname{ker} S \longrightarrow \overline{r a n S} \oplus(\overline{r a n S})^{\perp}$ have the matrix representations $\left(\begin{array}{cc}S_{1} & 0 \\ 0 & 0\end{array}\right)$ and $\left(\begin{array}{ll}X_{1} & X_{2} \\ X_{3} & X_{4}\end{array}\right)$.

Then

$$
\left\|\delta_{A, B}(X)+S\right\|=\left\|\left(\begin{array}{cr}
\delta_{A_{1}, B_{1}}\left(X_{1}\right)+S_{1} & * \\
* & *
\end{array}\right)\right\| \geq\left\|\delta_{A_{1}, B_{1}}\left(X_{1}\right)+S_{1}\right\|=\|S\|,
$$

which completes the proof of the theorem.

Next, we prove some commutativity results. Al-Moadjil [1] proved that if $N$ is a normal operator such that $N^{2} X=X N^{2}$ and $N^{3} X=X N^{3}$ for some $X \in \mathbf{B}(\mathscr{H})$, then $N X=X N$. Kittaneh [7] generalize this results for subnormal operators by taking $A$ and $B^{*}$ subnormal operators. This result was also generalized by Bachir [4] by taking $A$ is a dominant operator and $B^{*}$ is $P$-hyponormal operators. In this note, we generalize this result for any pair $\left(A, B^{*}\right)$ satisfying the Fuglede-Putnam Property.

Theorem 2.4. Let $A, B, X \in \mathbf{B}(\mathscr{H})$ and let the pair of operators $\left(A, B^{*}\right)$ satisfies the FugledePutnam Property. If $A^{2} X=X B^{2}$ and $A^{3} X=X B^{3}$, then $A X=X B$.

Proof. Let $Y=A X-X B$, then

$$
\begin{aligned}
& A^{2} Y=A^{3} X-A^{2} X B=X B^{3}-X B^{3}=0, \\
& Y B^{2}=A X B^{2}-X B^{3}=A^{3} X-A^{3} X=0,
\end{aligned}
$$

and

$$
A Y B=A^{2} X B-A X B^{2}=X B^{3}=A^{3} X=0 .
$$

Hence $A(A Y-Y B)=A^{2} Y-A Y B=0$ and $(A Y-Y B) B=A Y B-Y B^{2}=0$.

This yields that $A Y-Y B \in \operatorname{ker}\left(\delta_{A, B}\right) \cap \operatorname{ran}\left(\delta_{A, B}\right)=\{0\}$, therefore $A Y-Y B=0$. Hence $Y \in$ 
$\operatorname{ker}\left(\delta_{A, B}\right) \cap \operatorname{ran}\left(\delta_{A, B}\right)=\{0\}$ is obtained by Theorem 2.3, this implies that $Y=0$. That is, $A X=$ $X B$.

Kittaneh [7] proved that if $A, B \in \mathbf{B}(\not{H})$ such that $A^{2}=B^{2}, A^{3}=B^{3}, \operatorname{ker} A \subset \operatorname{ker} A^{*}$ and $\operatorname{ker} B \subset \operatorname{ker} B^{*}$, then $A=B$. This results can be generalized to any pair $\left(A, B^{*}\right)$ that satisfies the Fuglede-Putnam Property as follows.

Corollary 2.5. Let $A, B \in \mathbf{B}(\mathcal{H})$. If the pair of operators $\left(A, B^{*}\right)$ satisfies the Fuglede-Putnam Property and $A^{2}=B^{2}$ and $A^{3}=B^{3}$, then $A=B$.

Proof. This is an immediate consequence of Theorem 2.3 and Theorem 2.4.

Remark 2.6. Algebraic manipulations and induction show that the powers 2 and 3 in Theorem 2.4 and Corollary 2.5 can be replaced by any two relatively prime powers $n$ and $m$.

In order to obtain our next result, we require some lemmas.

Lemma 2.7. Let $T=\left(\begin{array}{cc}T_{1} & S \\ 0 & T_{2}\end{array}\right)$ be a quasihyponormal operator on $\not{H}=M \oplus M^{\perp}$, where $M$ is a $T$-invariant such that the restriction $T_{1}=\left.T\right|_{M}$ is normal. Then the range of $S$ is included in $\operatorname{ker} T_{1}$. In particular, if $T$ is injective, every normal part of $T$ reduces $T$.

Proof. Let $P$ be the orthogonal projection onto $M$. Then we have

$$
\begin{array}{rlrl}
P T^{*} T P=\left(\begin{array}{cc}
T_{1}^{*} T_{1} & 0 \\
0 & 0
\end{array}\right) & \leq P\left|T^{2}\right| P & & \text { (since } T \text { is quasihyponormal) } \\
& \leq\left(\begin{array}{cc}
\left(T_{1}^{* 2} T_{1}^{2}\right)^{\frac{1}{2}} & 0 \\
0 & 0
\end{array}\right) & \text { (by Hansen's inequality) } \\
& =\left(\begin{array}{cc}
T_{1}^{*} T_{1} & 0 \\
0 & 0
\end{array}\right) & & \text { (since } T_{1} \text { is normal). }
\end{array}
$$

Let $\left|T^{2}\right|=\left(\begin{array}{cc}X & Y \\ Y^{*} & Z\end{array}\right)$ be the matrix representation of $\left|T^{2}\right|$ on $H=M \oplus M^{\perp}$. Then we have $X=$ $T_{1}^{*} T_{1}$.

Since

$$
\left|T^{2}\right|^{2}=T^{2 *} T^{2} \quad=\left(\begin{array}{cc}
X^{2}+Y Y^{*} & X Y+Y Z \\
Z Y^{*}+Y^{*} X & Y^{*} Y+Z^{2}
\end{array}\right)=\left(\begin{array}{cc}
T_{1}^{* 2} T_{1}^{2} & T_{1}^{* 2} T_{1} S \\
S^{*} T_{1}^{*} T_{1}^{2} S^{*} S+T_{2}^{* 2} T_{2}^{2}
\end{array}\right) .
$$

Hence

$$
X^{2}+Y Y^{*}=T_{1}^{* 2} T_{1}^{2}=\left(T_{1}^{*} T_{1}\right)^{2}=X^{2} .
$$

This implies that $Y=0$. Thus we have

$$
\left|T^{2}\right|=\left(\begin{array}{cc}
\left.T_{1}^{*} T_{1}\right) & 0 \\
0 & Z^{2}
\end{array}\right) \quad \geq T^{*} T=\left(\begin{array}{cc}
\left.T_{1}^{*} T_{1}\right) & T_{1}^{*} S \\
S^{*} T_{1} & \left.S^{*} S+T_{2}^{*} T_{2}\right)
\end{array}\right),
$$


and hence $T_{1}^{*} S=0$. Thus the range of $S$ is included in $\operatorname{ker} T_{1}^{*}=\operatorname{ker} T_{1}$. If $T$ is injective, then so is $T_{1}$. Thus the second statement of lemma follows from [6, Lemma 10].

Lemma 2.8. If $T$ is a quasihyponormal operator, then every normal part of $T$ reduces $T$.

Proof. If $T$ is invertible, then $T$ is hyponormal. Hence the assertion holds by Stampfli result [12]. Now, we assume that $T$ is not invertible. Let $M$ be a normal part of $T$. By Lemma 2.7 $T$ is of the form $\left(\begin{array}{cc}N & S \\ 0 & 0\end{array}\right)$ on $M \oplus M^{\perp}$, where $N$ is normal and $r a n S \subset \operatorname{ker} N$. It is easy to see that

$$
T^{2 *} T^{2}=\left(\begin{array}{cc}
N^{2 *} N^{2} & N^{2 *} N S \\
S^{*} N^{*} N^{2} & S^{*} N^{*} N S
\end{array}\right)
$$

and

$$
\left(T^{*} T\right)^{2}=\left(\begin{array}{cc}
\left(N^{*} N\right)^{2}+N^{*} S S^{*} N & N^{*} N N^{*} S+N^{*} S S^{*} S \\
S^{*} N N^{*} N+S^{*} S S^{*} N & S^{*} N N^{*} S+S^{*} S S^{*} S
\end{array}\right)
$$

Then

$$
0 \leq T^{2 *} T^{2}-\left(T^{*} T\right)^{2}=\left(\begin{array}{ll}
-N^{*} S S^{*} N & -N^{*} S S^{*} S \\
-S^{*} S S^{*} N & -S^{*} S S^{*} S
\end{array}\right) .
$$

This implies that $S=0$.

Theorem 2.9. Let $T \in \mathbf{B}(\mathscr{H})$ be a quasihyponormal operator. Let $L \in \mathbf{B}(\mathscr{H})$ be self-adjoint which satisfies $T L=L T^{*}$. Then $T^{*} L=L T$.

Proof. First, we will show that If $T L=L T^{*}=0$, then $T^{*} L=L T=0$. Since $\operatorname{ker} T$ reduces $T, T L=0$ implies that $\operatorname{ran} L \subset \operatorname{ker} T \subset \operatorname{ker} T^{*}$. Hence $\overline{\operatorname{ran} T} \subset \operatorname{ker} T$. Therefore we have $T^{*} L=$ $L T=0$

Next, we prove the case $T L \neq 0$. Assume that $T$ is quasihyponormal. Using the decomposition $\mathscr{H}=\overline{\operatorname{ran} L} \oplus \operatorname{ker} L$, the operators $L$ and $T$ can be represented as follows.

$$
L=\left(\begin{array}{rr}
L_{1} & 0 \\
0 & 0
\end{array}\right), T=\left(\begin{array}{cc}
T_{1} & S \\
0 & T_{2}
\end{array}\right),
$$

where $L_{1}$ is self-adjoint with $\operatorname{ker} L_{1}=\{0\}$ and $T_{1}$ is also quasihyponormal. The assumption $T L=L T^{*}$ implies that $T_{1} L_{1}=L_{1} T_{1}^{*}$. Since $\operatorname{ker} T_{1}$ reduces $T_{1}$ and $L_{1}$, they are of the form $T_{1}=$ $T_{11} \oplus 0$ and $L_{1}=L_{11} \oplus L_{22}$ on $\overline{r a n L}=\overline{\operatorname{ker}|T|} \oplus \operatorname{ker} T_{1}$. Hence $T_{11}$ is an injective quasihyponormal operator and $L_{11}$ is self-adjoint operator which satisfies $T_{11} L_{11}=L_{11} T_{11}^{*}$. But this implies that $T_{11}$ is normal. Hence $T_{1}=T_{11} \oplus 0$ is also normal. By Fuglede-Putnam Theorem, we see that $T_{1}^{*} L_{1}=L_{1} T_{1}$. since $T_{1}$ is normal, $S=0$, so we have $T^{*} L=L T$.

Corollary 2.10. Let $T \in \mathbf{B}(\mathscr{H})$ be a quasihyponormal operator. If $X \in \mathbf{B}(\not \mathscr{\ell})$ and $T X=X T^{*}$, then $T^{*} X=X T$.

Proof. Let $X=Y+i Z$ be the cartesian decomposition of $X$. Then $T X=X T^{*}$ implies that $T Y=Y T^{*}$ and $T Z=Z T^{*}$. By Theorem 2.9, we have $T^{*} Y=Y T$ and $T^{*} Z=Z T$. Hence $T X=X T^{*}$. 
Corollary 2.11. Let $T, S \in \mathbf{B}(\mathscr{H})$ be such that $T$ and $S^{*}$ are quasihyponormal operators. If $X \in \mathbf{B}(\mathscr{H})$ and $T X=X S$, then $T^{*} X=X S^{*}$.

Proof. Let $A=\left(\begin{array}{ll}T & 0 \\ 0 & S^{*}\end{array}\right)$ and $B=\left(\begin{array}{ll}0 & X \\ 0 & 0\end{array}\right)$ on $\mathscr{H} \oplus \mathscr{H}$. Then $A$ is quasihyponormal operator on $\mathscr{H} \oplus \mathscr{H}$ that satisfies

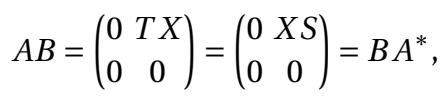

by Corollary 2.10, we have $A^{*} B=B A$ and therefore $T^{*} X=X S^{*}$.

The most recent generalization of the Fuglede-Putnam theorem was obtained by Uchiyama and Tanahashi [15] and can be stated as follows.

Theorem 2.12. Let $T, S \in \mathbf{B}(\mathscr{H})$ be such that $T$ and $S^{*}$ are $p$-hyponormal operators. If $X \in \mathbf{B}(\mathscr{H})$ and $T X=X S$, then $T^{*} X=X S^{*}$.

As an application of the above results we have

Lemma 2.13. Let $V, A$ and $X$ be operators $\operatorname{in} \mathbf{B}(\not{H})$. If $V$ is an isometry, $A^{*}$ is $p$-hyponormal, and $X$ is one-one, then $V X=X A$ implies $A$ is unitary.

Proof. By Corollary 11, $V X=X A$ implies that $V^{*} X=X A^{*}$. Multiply the first equation on the left by $V^{*}$ to get $X=V^{*} A^{*} A$. Therefore, $X=X A^{*} A$. Let $X=U P$ be the polar decomposition of $X$, then $U$ is unitary and $P$ is one-one. But this implies that $1=A^{*} A$. Since $A^{*}$ is $p$-hyponormal and $A^{*} A=1$, it follows that $A$ is normal and hence unitary.

An attempt to generalize Theorem [7, Theorem 1] to the hyponormal case was made by T. Furuta [5], who obtained the following result.

Theorem 2.14. If $A$ and $B^{*}$ are hyponormal operators in $\mathbf{B}(\mathscr{H})$, then for any $X \in \mathscr{C}_{2}, A X-$ $X B \in \mathscr{C}_{2}$ implies $A^{*} X-X B^{*} \in \mathscr{C}_{2}$.

In the following theorem, we relax the hypotheses on $A$ and $B^{*}$ in Theorem 2.14 to $p$ hyponormality.

Theorem 2.15. Let $A, B \in \mathbf{B}(\mathscr{H})$ be such that $A, B^{*}$ are $p$-hyponormal. Then for any $X \in \mathscr{C}_{2}$, $A X-X B \in \mathscr{C}_{2}$ implies $A^{*} X-X B^{*} \in \mathscr{C}_{2}$.

Proof. Let $A, B^{*}$ be $p$-hyponormal for $p \geq \frac{1}{2}$ and let $U|B|$ be the the polar decomposition of $B$. Then it follows from [2] that the Aluthge transform $\widetilde{B^{*}}$ of $B$ is hyponormal and satisfies

$$
|\widetilde{B}| \leq|B| \leq\left|\widetilde{B^{*}}\right|
$$

and

$$
A Y-Y \widetilde{B} \in \mathscr{C}_{2}
$$


, where $Y=X U|B|^{\frac{1}{2}}$. Using the decomposition $\mathscr{H}=\operatorname{ker} Y^{\perp} \oplus \operatorname{ker} Y$, we see that $A, \widetilde{B}, Y$ are of the form

$$
A=\left(\begin{array}{cc}
A_{1} & T \\
0 & A_{2}
\end{array}\right), \widetilde{B}=\left(\begin{array}{cc}
B_{1} & 0 \\
S & B_{2}
\end{array}\right), Y=\left(\begin{array}{cc}
Y_{1} & 0 \\
0 & 0
\end{array}\right),
$$

where, $A_{1}$ is $p$-hyponormal, $B_{1}^{*}$ is hyponormal and $Y_{1}$ is one-one mapping with dense range. It follows from equation 1.2 that

$$
A_{1} Y_{1}-Y_{1} B_{1} \in \mathscr{C}_{2} .
$$

Hence $A_{1}, B_{1}$ are normal by [11, Theorem 10], so that $T=0$ by [15, Lemma 12] and $S=0$ by [6]. Thus $|B|=\left|B_{1}\right| \oplus J$, for some positive operator $J$, by equation 1.1 and [15, Lemma13] that $U=\left(\begin{array}{cc}U_{11} & U_{12} \\ 0 & U_{22}\end{array}\right)$. Let $X=\left(\begin{array}{ll}X_{11} & X_{12} \\ X_{21} & X_{22}\end{array}\right)$ be a $2 \times 2$ matrix representation of $X$ with respect to the decomposition $\mathscr{H}=\operatorname{ker} Y^{\perp} \oplus \operatorname{ker} Y$. Then, $Y=X U|B|^{\frac{1}{2}}$ implies that $Y_{1}=X_{11} U_{11}\left|B_{1}\right|^{\frac{1}{2}}$ and hence $\operatorname{ker} B_{1} \subset \operatorname{ker} Y_{1}=\{0\}$. This shows that $B_{1}$ is one-one (hence, it has dense range), so that $U_{12}=0$ and $B=B_{1} \oplus B_{3}$, for some co- $p$-hyponormal operator $B_{3}$ by [15, Lemma 13]. Since,

$$
\left(\begin{array}{ll}
Y_{1} & 0 \\
0 & 0
\end{array}\right)=Y=X U|B|^{\frac{1}{2}}=\left(\begin{array}{ll}
X_{11} & X_{12} \\
X_{21} & X_{22}
\end{array}\right)\left(\begin{array}{cc}
U_{11}\left|B_{1}\right|^{\frac{1}{2}} & 0 \\
0 & U_{22}\left|A_{33}\right|^{\frac{1}{2}}
\end{array}\right),
$$

we have the following statements.

$X_{12} U_{22}\left|B_{3}\right|^{\frac{1}{2}}=0$; hence $X_{12} B_{3}=0$ because $B_{3}=U_{22}\left|B_{3}\right|$.

$X_{12} U_{11}\left|B_{1}\right|^{\frac{1}{2}}=0$; hence $X_{12}=0$ because $U_{11}\left|B_{1}\right|^{\frac{1}{2}}$ has dense range.

$X_{22} U_{22}\left|B_{3}\right|^{\frac{1}{2}}=0$; hence $X_{22} B_{3}=0$.

The assumption $A X-X B \in \mathscr{C}_{2}$ imply that

$A_{1} X_{11}-X_{11} B_{1} \in \mathscr{C}_{2}, X_{12} B_{3}=A_{1} X_{12}=0$ and $X_{22} B_{3}=A_{2} X_{22}=0$. Since $A_{1}$ and $B_{1}$ are normal we have $A_{1}^{*} X_{11}-X_{11} B_{1}^{*} \in \mathscr{C}_{2}$, by Fuglede-Putnam Theorem. The $p$-hyponormality of $B_{3}^{*}$ shows that $\operatorname{ran}_{3}^{*} \subset \overline{B_{3}}$. Also we have $\operatorname{ker} A_{2} \subset \operatorname{ker} A_{2}^{*}$ from the fact $A_{2}$ is $p$-hyponormal. Hence, we also have $X_{12} B_{3}^{*}=A_{1}^{*} X_{12}=0$ and $X_{22} B_{3}^{*}=A_{2}^{*} X_{22}=0$. This implies that $A X-X B \in \mathscr{C}_{2}$.

Next, we prove the case where $0<p \leq \frac{1}{2}$. Let $Y$ be as above. Then $\widetilde{B^{*}}$ is $p+\frac{1}{2}$-hyponormal and satisfies $A X-X \widetilde{B} \in \mathscr{C}_{2}$. Use the same argument as above. We obtain $\widetilde{B}=B_{1} \oplus B_{2}$ on $\mathscr{H}=$ $\operatorname{ker} Y^{\perp} \oplus \operatorname{ker} Y$ and $A=A_{1} \oplus A_{2}$, where $B_{1}$ is an injective normal operator and $A_{1}$ is also normal. Hence, we have $B=B_{1} \oplus B_{3}$ for some $p$-hyponormal $B_{3}^{*}$. Again using the same argument as above we obtain the result.

\section{References}

[1] A. Al-Moadjil, On the commutant of relatively prime powers in banach algebra, Proc. Amer. Math. Soc. 57(1976), 243-249.

[2] A. Aluthge, On the $p$-hyponormal for $0<p<\frac{1}{2}$, Integ. Equat. Oper. Th. 13(1990), 307-315.

[3] J. Anderson, On normal derivations, Proc. Amer. Math. Soc. 38(1973), 135-140.

[4] A. Bachir, Generalized derivation, SUT J. Math 40(2004), no.2, 111-114.

[5] T. Furuta, An extension of the Fuglede-Putnam Theorem to subnormal operators using a HilbertSchmidt norm inequality, Proc. Amer. Math. Soc.81(1981), 240-242. 
[6] I. Kim, The Fuglede-Putnam Theorem for $(p, k)$-quasihyponormal operators, J. Ineq. Appl. Artical 47481(2006), 1-7.

[7] F. Kittaneh, On generalized Fuglede-Putnam Theorems of Hilbert-Schmidt type, Proc. Amer. Math. Soc. 88(1983), 293-298.

[8] F. Kittaneh, Inequalities for the schatten p-norm II, Glasgow Math. J. 29(1987), 99-104.

[9] F. Kittaneh, On normal derivations of Hilbert-Schmidt type, Glasgow Math. J. 29(1987), 245-248.

[10] F. Kittaneh, Normal derivations in normal ideals, Proc. Amer. Math. Soc., 123(1995), 1179-1785.

[11] M. Otieno, On intertwining and $\omega$-hyponormal operators, Opuscula Math. 25(2005), 275-285.

[12] J. G. Stampfli, Hyponormal operators, Pacific J. Math., 12(1962), 1453-1458.

[13] J. Stampfli and B. Wadhwa, On dominant operators, Monatshefte. Für. Math. 84(1977), 33-36.

[14] T. Tanahashi, On log-hyponormal operators, Math. Ineq. Appl., 2(1999), 123-130.

[15] A. Uchiyama and K. Tanahashi, Fuglede-Putnam Theorem For p-hyponormal Or log-hyponormal operators, Glassgow Math. J. 44(2002), 397-410.

School of Mathematical Sciences, Faculty of Science and Technology, Universiti Kebangsaan Malaysia, 43600 UKM, Selangor, Malaysia.

E-mail:malik_okasha@yahoo.com,msn@pkrisc.cc.ukm.my, shabir@pkrisc.cc.ukm.my 